\title{
A LINEARIZATION OF THE CIRCULAR MAXIMAL OPERATOR
}

\author{
DANIEL M. OBERLIN
}

(Communicated by J. Marshall Ash)

\begin{abstract}
An interesting linearization of the circular maximal operator is of restricted weak type $(2,2)$.
\end{abstract}

The spherical maximal operator $\mathscr{M}$ on $\mathbf{R}^{n}$ is defined by

$$
\mathscr{M} f(x)=\sup _{t>0} \int_{|y|=1}|f|(x-t y) d y .
$$

Here $f$ is an appropriate function and $d y$ denotes normalized Lebesgue measure on the unit sphere in $\mathbf{R}^{n}$. In [4] Stein proved that $\mathscr{M}$ is bounded on $L^{p}\left(\mathbf{R}^{n}\right)$ if $p>n /(n-1)$ and $n \geq 3$. More recently Bourgain [1] established the same result for $n=2$. Bourgain [2] also noted that when $n \geq 3$ and $p=n /(n-1), \mathscr{M}$ is restricted weak type $(p, p)$-that is, $\mathscr{M}$ maps $L^{p, 1}\left(\mathbf{R}^{n}\right)$ into $L^{p, \infty}\left(\mathbf{R}^{n}\right)$. This result implies that of [4]. It is then natural to ask if $\mathscr{M}$ maps $L^{2,1}\left(\mathbf{R}^{2}\right)$ into $L^{2, \infty}\left(\mathbf{R}^{2}\right)$. Leckband [3] provided a partial result: the answer is yes if one restricts to the subspace of radial functions. The purpose of this note is to give a partial result with a different flavor. We restrict the operator instead of its domain and define, as in [1, p. 70], a linearization $T$ of $\mathscr{M}$ by

$$
T f(x)=\int_{|y|=1} f(x-|x| y) d y .
$$

Theorem. The operator $T$ maps $L^{2,1}\left(\mathbf{R}^{2}\right)$ into $L^{2, \infty}\left(\mathbf{R}^{2}\right)$.

In all known cases the mapping properties of $T$ are as bad as those of $\mathscr{M}$, and so this theorem lends support to the conjecture that $\mathscr{M}$ is of restricted weak type $(2,2)$ on $\mathbf{R}^{2}$.

For $x \in \mathbf{R}^{2}$, let $L_{x}$ be the line through $x$ perpendicular to the radial segment from the origin to $x$. Define an operator $S$ by

$$
S f(x)=\frac{1}{|x|} \int_{L_{x}} f(y) d y,
$$

Received by the editors April 14, 1988.

1980 Mathematics Subject Classification (1985 Revision). Primary 42B25.

Partially supported by a grant from the National Science Foundation. 
where $d y$ is one-dimensional Lebesgue measure on $L_{x}$. Then $S$ is a weighted version of the Radon transform on $\mathbf{R}^{2}$ and (see Lemma 2) is equivalent to the adjoint of $T$. Our theorem follows from the boundedness of $S$ from $L^{2,1}\left(\mathbf{R}^{2}\right)$ to $L^{2, \infty}\left(\mathbf{R}^{2}\right)$, which is proved as Lemma 4 . To simplify notation we regard points in $\mathbf{R}^{2}$ as complex numbers.

Lemma 1. If $z \neq 0$ is a complex number, define $z^{\prime}=z e^{-i \pi / 2} /|z|$. If $g$ and $h$ are nonnegative and measurable, then

$$
\begin{gathered}
\int_{0}^{2 \pi} \int_{0}^{2 \pi} h\left(e^{i \theta}+e^{i t}\right) g\left(e^{i \theta}\right) d t d \theta \\
=\int_{|z|<2} h(z)\left[g\left(\frac{z}{2}+z^{\prime} \sqrt{1-\frac{|z|^{2}}{4}}\right)+g\left(\frac{z}{2}-z^{\prime} \sqrt{1-\frac{|z|^{2}}{4}}\right)\right] \frac{d z}{|z| \sqrt{1-\frac{|z|^{2}}{4}}},
\end{gathered}
$$

where $d z$ denotes two-dimensional Lebesgue measure.

Proof. If $z=e^{i \theta}+e^{i t}$ with $\theta<t<\theta+\pi$, then a sketch shows that

$$
e^{i \theta}=\frac{z}{2}+z^{\prime} \sqrt{1-\frac{|z|^{2}}{4}} .
$$

Also the Jacobian of the map

$$
(t, \theta) \mapsto z=e^{i \theta}+e^{i t}
$$

is

$$
|\sin (t-\theta)|=|z| \sqrt{1-\frac{|z|^{2}}{4}}
$$

Thus the formula

$$
\begin{aligned}
\int_{0}^{2 \pi} & \int_{\theta}^{\theta+\pi} h\left(e^{i \theta}+e^{i t}\right) g\left(e^{i t}\right) d t d \theta \\
& =\int_{|=|<2} h(z) g\left(\frac{z}{2}+z^{\prime} \sqrt{1-\frac{|z|^{2}}{4}}\right) \frac{d z}{|z| \sqrt{1-\frac{|z|^{2}}{4}}}
\end{aligned}
$$

is just a change of variable. A similar formula for the range $\theta+\pi<t<\theta+2 \pi$ completes the proof.

Lemma 2. If $g$ and $h$ are nonnegative and measurable, then

$$
\int_{\mathbf{R}^{2}} T f(x) g(x) d x=\frac{1}{2} \int_{\mathbf{R}^{2}} f(x) S g\left(\frac{x}{2}\right) d x .
$$


Proof.

$$
\begin{aligned}
\int_{\mathbf{R}^{2}} T f(x) g(x) d x=\int_{0}^{\infty} \int_{0}^{2 \pi} \int_{0}^{2 \pi} f\left(r e^{i \theta}+r e^{i t}\right) g\left(r e^{i \theta}\right) r d t d \theta d r \\
=\int_{0}^{\infty} \int_{0}^{2} \int_{0}^{2 \pi} f\left(r u e^{i \phi}\right)\left\{g\left(r\left[\frac{u e^{i \phi}}{2}+e^{i\left(\phi-\frac{\pi}{2}\right)} \sqrt{1-\frac{u^{2}}{4}}\right]\right)\right. \\
\left.+g\left(r\left[\frac{u e^{i \phi}}{2}-e^{i\left(\phi-\frac{\pi}{2}\right)} \sqrt{1-\frac{u^{2}}{4}}\right]\right)\right\} \frac{r d \phi d u d r}{\sqrt{1-\frac{u^{2}}{4}}}
\end{aligned}
$$

by Lemma 1, with $z=e u^{i \phi}$. To this last expression apply Fubini's theorem, let $s=u r$, and apply Fubini's theorem again. The result is

$$
\begin{array}{r}
\int_{0}^{\infty} \int_{0}^{2} \int_{0}^{2 \pi} f\left(s e^{i \phi}\right)\left\{g\left(\frac{s}{2} e^{i \phi}+\frac{s}{u} e^{i\left(\phi-\frac{\pi}{2}\right)} \sqrt{1-\frac{u^{2}}{4}}\right)\right. \\
\left.+g\left(\frac{s}{2} e^{i \phi}-\frac{s}{u} e^{i\left(\phi-\frac{\pi}{2}\right)} \sqrt{1-\frac{u^{2}}{4}}\right)\right\} \frac{s d \phi d u d s}{u^{2} \sqrt{1-\frac{u^{2}}{4}}} \\
=\int_{0}^{\infty} \int_{0}^{\infty} \int_{0}^{2 \pi} f\left(s e^{i \phi}\right)\left\{g\left(\frac{s}{2} e^{i \phi}+s v e^{i\left(\phi-\frac{\pi}{2}\right)}\right)\right. \\
\left.\quad+g\left(\frac{s}{2} e^{i \phi}-s v e^{i\left(\phi-\frac{\pi}{2}\right)}\right)\right\} s d \phi d v d s \\
=\int_{0}^{\infty} \int_{0}^{\infty} \int_{0}^{2 \pi} f\left(s e^{i \phi}\right)\left\{g\left(\frac{s}{2} e^{i \phi}+t e^{i\left(\phi-\frac{\pi}{2}\right)}\right)\right. \\
\left.+g\left(\frac{s}{2} e^{i \phi}-t e^{i\left(\phi-\frac{\pi}{2}\right)}\right)\right\} d \phi d t d s
\end{array}
$$

where the equalities are from the changes of variable

$$
v=\frac{\sqrt{1-\frac{u^{2}}{4}}}{u} \text { and } t=s v \text {. }
$$

This last integral is

$$
\frac{1}{2} \int_{\mathbf{R}^{2}} f(x) S g\left(\frac{x}{2}\right) d x .
$$

In what follows, $|\cdot|$ will denote Lebesgue measure on either $\mathbf{R}$ or $\mathbf{R}^{2}$, the exact meaning being clear from the context. Also, $\chi(x, E)$ will stand for the characteristic function of the set $E$ evaluated at $x$.

Lemma 3. Suppose $h$ and $k$ are measurable functions on $[0, \infty]$ with $0 \leq$ $h, k \leq 1$. Then

$$
\int_{0}^{\infty} \int_{0}^{\infty} \min (h(s), k(r)) d s d r \leq 4\left[\int_{0}^{\infty} \operatorname{sh}(s) d s\right]^{\frac{1}{2}}\left[\int_{0}^{\infty} r k(r) d r\right]^{\frac{1}{2}} .
$$

Proof. The left hand side of the conclusion is not affected by measure-preserving rearrangements of $h$ and $k$ while the right hand side will be least when $h$ and $k$ 
are decreasing. So replacing $h$ and $k$ by suitable approximations shows that it is enough to establish the lemma under the additional hypotheses that $h$ and $k$ are continuous positive strictly decreasing functions satisfying $h(0)=k(0)=1$. Now

$$
\begin{aligned}
\int_{0}^{\infty} \int_{0}^{\infty} \min (h(s), k(r)) d s d r= & \int_{0}^{\infty} h(s)|\{r: k(r)>h(s)\}| d s \\
& +\int_{0}^{\infty} k(r)|\{s: h(s)>k(r)\}| d r=I_{1}+I_{2} .
\end{aligned}
$$

Since $|\{r: k(r)>h(s)\}|=k^{-1}(h(s))$,

$$
\begin{aligned}
I_{1} & =\int_{0}^{\infty} h(s) k^{-1}(h(s)) d s=\int_{0}^{\infty} \int_{0}^{k^{-1}(h(s))} \int_{0}^{k(x)} \chi(y,[0, h(s)]) d y d x d s \\
& =\int_{0}^{\infty} \int_{0}^{k(x)} \int_{0}^{\infty} \chi\left(x,\left[0, k^{-1}(h(s))\right]\right) \chi(y,[0, h(s)]) d s d y d x \\
& \leq \int_{0}^{\infty} \int_{0}^{k(x)} h^{-1}(y) d y d x \\
& =\int_{0}^{1} \int_{0}^{k^{-1}(y)} h^{-1}(y) d x d y=\int_{0}^{1} h^{-1}(y) k^{-1}(y) d y \\
& \leq\left(\int_{0}^{1}\left[h^{-1}(y)\right]^{2} d y\right)^{1 / 2}\left(\int_{0}^{1}\left[k^{-1}(y)\right]^{2} d y\right)^{1 / 2} \\
& =2\left[\int_{0}^{\infty} \operatorname{sh}(s) d s\right]^{1 / 2}\left[\int_{0}^{\infty} r k(r) d r\right]^{1 / 2},
\end{aligned}
$$

where the last equality can be verified by comparing two methods for computing the volume of a solid of revolution. Now interchanging $h$ and $k$ completes the proof of the lemma.

Lemma 4. There is a positive number $C$ such that if $E$ and $F$ are measurable subsets of $\mathbf{R}^{2}$, then

$$
\int_{\mathbf{R}^{2}} \chi(x, E) S \chi(\cdot, F)(x) d x \leq C|E|^{1 / 2}|F|^{1 / 2} .
$$

Proof. The operator $S$ is given by the formula

$$
S g\left(r e^{i \theta}\right)=\frac{1}{r} \int_{-\infty}^{\infty} g\left(r e^{i \theta}+t e^{i\left(\theta+\frac{\pi}{2}\right)}\right) d t,
$$

so we will show that

$$
\int_{0}^{\infty} \int_{-\infty}^{\infty} \int_{0}^{2 \pi} \chi\left(r e^{i \theta}, E\right) \chi\left(r e^{i \theta}+t e^{i\left(\theta+\frac{\pi}{2}\right)}, F\right) d \theta d t d r \leq C|E|^{1 / 2}|F|^{1 / 2} .
$$

We will actually consider only the integral $\int_{0}^{\infty} \int_{0}^{\infty} \int_{0}^{2 \pi}$-the integral $\int_{0}^{\infty} \int_{-\infty}^{0} \int_{0}^{2 \pi}$ is treated analogously. The change of variable

$$
x=x_{r}(\theta, t)=r e^{i \theta}+t e^{i\left(\theta+\frac{\pi}{2}\right)}
$$


shows that

$$
\begin{aligned}
\int_{0}^{\infty} & \int_{0}^{\infty} \int_{0}^{2 \pi} \chi\left(r e^{i \theta}, E\right) \chi\left(r e^{i \theta}+t e^{i\left(\theta+\frac{\pi}{2}\right)}, F\right) d \theta d t d r \\
& =\int_{0}^{\infty} \int_{\{|x|>r\}} \chi(p(r, x), E) \chi(x, F) \frac{d x}{\sqrt{|x|^{2}-r^{2}}} d r
\end{aligned}
$$

where $p(r, x)$ is the point $r e^{i \theta}$ such that $x$ can be written $x=r e^{i \theta}+t e^{i\left(\theta+\frac{\pi}{2}\right)}$ for some $t>0$. We write the last integral as $I_{1}+I_{2}$ where

$$
I_{1}=\int_{0}^{\infty} \int_{\{4 r>|x|>r\}}, \quad I_{2}=\int_{0}^{\infty} \int_{\{|x|>4 r\}},
$$

and we begin by considering $I_{1}$ :

$$
\begin{aligned}
\int_{0}^{\infty} & \int_{\{4 r>|x|>r\}} \chi(p(r, x), E) \chi(x, F) \frac{d x}{\sqrt{|x|^{2}-r^{2}}} d r \\
\leq & C \int_{0}^{\infty}\left(r^{-\frac{1}{2}}|F \cap\{r<|x|<4 r\}|^{1 / 2}\right) \\
& \times\left(r^{\frac{1}{2}}\left\|\chi(p(r, x), E)\left(|x|^{2}-r^{2}\right)^{-\frac{1}{2}} \chi(|x|,(r, \infty))\right\|_{L_{x}^{2} \cdot \infty} d r,\right.
\end{aligned}
$$

since $L^{2, \infty}$ is the dual of $L^{2,1}$. Applying Hölder's inequality shows that this last integral is dominated by

$$
\begin{aligned}
C\left(\int_{0}^{\infty} \mid F\right. & \left.\cap\{r<|x|<4 r\} \mid \frac{d r}{r}\right)^{1 / 2} \\
& \times\left(\int_{0}^{\infty}\left\|\chi(p(r, x), E)\left(|x|^{2}-r^{2}\right)^{-\frac{1}{2}} \chi(|x|,(r, \infty))\right\|_{L_{x}^{2 . \infty}}^{2} r d r\right)^{1 / 2} .
\end{aligned}
$$

The first term in parentheses is just $(\log 4) \cdot|F|$. We will now prove that the second parenthesized integral is bounded by $C|E|$ and hence that $I_{1} \leq$ $C|E|^{1 / 2}|F|^{1 / 2}$. A sketch shows that if

$$
s e^{i \phi}=r e^{i \theta}+t e^{i\left(\theta+\frac{\pi}{2}\right)},
$$

then

$$
\exp (i \theta)=\exp \left(i\left[\phi-\cos ^{-1}(r / s)\right]\right)
$$

Thus

$$
\left|\left\{\phi \in[0,2 \pi): p\left(r, s e^{i \phi}\right) \in E\right\}\right|=\left|\left\{\phi \in[0,2 \pi): r e^{i \phi} \in E\right\}\right|,
$$

and so, for $\lambda>0$,

$$
\begin{aligned}
\left|\left\{x:|x|>r, p(r, x) \in E,\left(|x|^{2}-r^{2}\right)^{-\frac{1}{2}}>\lambda\right\}\right| \\
\quad=\int_{r}^{\sqrt{r^{2}+\lambda^{-2}}}\left|\left\{\phi \in[0,2 \pi): p\left(r, s e^{i \phi}\right) \in E\right\}\right| s d s \\
\quad=\left|\left\{\phi \in[0,2 \pi): r e^{i \phi} \in E\right\}\right| / 2 \lambda^{2} .
\end{aligned}
$$


It follows that

$$
\int_{0}^{\infty}\left\|\chi(p(r, x), E)\left(|x|^{2}-r^{2}\right)^{-\frac{1}{2}} \chi(|x|,(r, \infty))\right\|_{L_{x}^{2} \cdot \infty}^{2} r d r \leq C|E|
$$

as claimed. Thus the proof will be complete when we see that

$$
I_{2} \leq C|E|^{1 / 2}|F|^{1 / 2} \text {. }
$$

Now if $|x|>4 r$, then

$$
\frac{1}{\sqrt{|x|^{2}-r^{2}}} \leq \frac{2}{|x|}
$$

so

$$
\begin{gathered}
\frac{I_{2}}{4 \pi} \leq \int_{0}^{\infty} \int_{0}^{\infty} \frac{1}{2 \pi} \int_{0}^{2 \pi} \chi\left(p\left(r, s e^{i \phi}\right), E\right) \chi\left(s e^{i \phi}, F\right) d \phi d s d r \\
\leq \int_{0}^{\infty} \int_{0}^{\infty} \min \left\{\frac{1}{2 \pi} \int_{0}^{2 \pi} \chi\left(p\left(r, s e^{i \phi}\right), E\right) d \phi, \frac{1}{2 \pi} \int_{0}^{2 \pi} \chi\left(s e^{i \phi}, F\right) d \phi\right\} d s d r .
\end{gathered}
$$

As noted earlier,

$$
\int_{0}^{2 \pi} \chi\left(p\left(r, s e^{i \phi}\right), E\right) d \phi=\int_{0}^{2 \pi} \chi\left(r e^{i \phi}, E\right) d \phi .
$$

Thus an application of Lemma 3 completes the proof.

\section{REFERENCES}

1. J. Bourgain, Averages in the plane over convex curves and maximal operators, J. d'Analyse Math. 47 (1986), 69-85.

2. $\_$, Estimations de certaines functions maximales, C.R. Acad. Sci. Paris Sér. I 301 (1985), 499-502.

3. M. Leckband, A note on the spherical maximal operator for radial functions, Proc. Amer. Math. Soc. 100 (1987), 635-640.

4. E. Stein, Maximal functions: spherical means, Proc. Nat. Acad. Sci. U.S.A. 73 (1976), 2174-2175.

Department of Mathematics, Florida State University, Tallahassee, Florida 32306 